\title{
RELAÇÃO ENTRE QUALIDADE DE VIDA E ATIVIDADE FÍSICA: UMA REVISÃO SISTEMÁTICA DA LITERATURA NACIONAL
}

\author{
Deise Pelozatto, Rômulo Araújo Fernandes \\ Curso de Educação Física da UNOESTE, Presidente Prudente, SP.
}

\begin{abstract}
RESUMO
Acredita-se que a prática regular de atividade física tenha papel significativo na qualidade de vida de qualquer individuo, independentemente da idade, sexo e etnia. Nesse sentido, na literatura nacional, muita atenção tem sido dada ao assunto nos últimos anos. O objetivo deste estudo foi analisar, por meio de uma revisão sistemática de literatura, as informações nacionais acerca da relação entre qualidade de vida e atividade física, bem como, apontar direções para futuros estudos. O estudo constituiu-se de uma revisão sistemática da literatura nacional, para a qual foi utilizada a base de dados da Scientific Electronic Library Online (SCIELO). No levantamento de dados foram usados apenas artigos originais, conduzidos com população brasileira. A análise estatística foi conduzida com o uso do teste de sinal e o teste exato de Fisher, sendo a significância estatística fixada em $p<5 \%$. Em todos os estudos analisados $(n=6 ; 100 \%)$, 0 tamanho das amostras envolvidas nos estudos variou de 30 a 863 sujeitos. A maioria dos trabalhos foi publicada nos dois últimos anos, havendo maior ocorrência de estudos envolvendo idosos (66,7\%; $\mathrm{p}=0,404)$. Os dois únicos instrumentos usados para quantificar a qualidade de vida foram os questionários: SF-36 e o World Health Organization Quality of Life (WHOQOL). A maioria dos estudos avaliou mulheres e poucos avaliaram homens. Independente da idade, em todos os estudos analisados houve interação entre maior prática de atividade física e assim melhor escores nos domínios da qualidade de vida. Concluiu-se que a qualidade de vida parece estar associada positivamente com a prática de atividade física independentemente da idade, porém, mais estudos são necessários.
\end{abstract}

Palavras-chave: Qualidade de vida, adultos, atividade física, Brasil.

\section{RELATIONSHIP BETWEEN QUALITY OF LIFE AND PHYSICAL ACTIVITY: A SYSTEMATIC REVIEW OF NATIONAL LITERATURE}

\begin{abstract}
It is possible that physical activity practice affects the quality of life of any subject, independently of age, sex and ethnicity. Therefore, this issue has received increasing attention. The aim of this study was to analyze through systematic review, national data about the relationship between physical activity and quality of life, as well as, to indicate future lines of research. It was carried out a systematic review in the Scientific Electronic Library Online (SCIELO). Only Brazilian original articles were taken into count. Statistical analysis was composed by signal test and Fisher's exact test, being significance set at $p<5 \%$. In all analyzed studies $(n=6 ; 100 \%)$, sample size ranged from 30 to 863 subjects. Higher number of articles was published in last two years, and many analyses involved elderly (66.7\%; $p=0.404)$. Quality of life was measured by two questionnaires: SF-36 and World Health Organization Quality of Life (WHOQOL). There was less articles analyzing men, comparing with women. In all analyzed articles, physical activity was positively related to better quality of life, independently of age. In summary, quality of life seems positively related to higher physical activity independently of age, however, more studies are necessary.
\end{abstract}

Keywords: Quality of life, adults, physical activity, Brazil 


\section{INTRODUÇÃO}

Ao longo das últimas décadas, o estudo da qualidade de vida tornou-se foco de crescente interesse por parte de pesquisadores de diferentes áreas do conhecimento, caso da Educação Física. De fato, alguns estudos têm indicado que pessoas mais ativas fisicamente apresentam melhores escores de qualidade de vida (SGUIZZATTO et al., 2006) e, dessa forma, nos leva a gerar a hipótese de que a promoção da atividade física na população em geral possa trazer melhoras neste sentido também.

Por outro lado, muito pouco se sabe ainda sobre o comportamento de tal variável em diferentes grupos etários, ou seja, embora existam estudos com adultos e idosos (SGUIZZATTO et al., 2006; MENESES et al., 2011), ainda são poucas as investigações analisando a relação da atividade física com a qualidade de vida envolvendo grupos como crianças e adolescentes. No caso de crianças e adolescentes, grupos que passam por diferentes mudanças físicas e comportamentais, a qualidade de vida pode representar um importante indicador de sinais de depressão, dentre outras condições e, dessa forma, a prática de atividades físicas pode assumir papel de destaque em tais populações (GORDIA et al., 2010). Outro fator que também deve ser levado em consideração é a influencia do sexo. Sabe-se que, dependendo do domínio analisado, homens e mulheres apresentam padrões de prática de atividade física distintos, porém, não se sabe os efeitos de cada um destes domínios sobre a qualidade de vida, bem como, não se sabe ao certo se o efeito é similar em ambos os sexos.

Nesse sentido, faz-se necessário averiguar em que direções têm avançado o estudo da relação qualidade de vida e atividade física, bem como, indicar lacunas que precisam ser preenchidas na busca de um entendimento maior sobre esta variável que se mostra tão promissora.

Assim, o objetivo do presente estudo foi analisar, utilizando um modelo de revisão sistemática da literatura nacional, os dados existentes acerca da relação qualidade de vida e atividade física em diferentes grupos populacionais brasileiros.

\section{MÉTODOS}

A revisão sistemática da literatura foi realizada em uma base de dados eletrônica e, dessa forma, não foi feita busca manual por documentos ou por meio de contato com autores. Assim, por ser a maior base nacional de periódicos científicos, a busca eletrônica foi realiza na Scientific Electronic Library Online (SCIELO), a qual disponibiliza todos os seus artigos na íntegra e sem custos. Ressalta-se que não foi feita busca em outras revistas da área de Educação Física não indexadas na base SCIELO.

Foram utilizadas as seguintes palavraschaves: qualidade de vida, atividade física, atividade motora, exercício físico. Não foi feita busca de artigos utilizando os descritores correspondentes em língua inglesa ou espanhola.

Dentre os artigos detectados, uma análise inicial foi realizada com bases nos títulos, se dúvidas existissem, os resumos dos mesmos foram analisados para alcançar a certeza de que os artigos se enquadravam nos critérios de inclusão estabelecidos: (i) estudo original de delineamento transversal, retrospectivo ou prospectivo; (ii) estudo realizado no Brasil; (iii) não ser estudo de revisão. Após a leitura do título e análise do resumo (quando necessário), os artigos selecionados foram obtidos na integra e posteriormente examinados, buscando o arquivamento das variáveis independentes envolvidas no estudo (sexo dos participantes, idade dos participantes, tipo de instrumento utilizado para avaliar qualidade de vida). Uma 
planilha inicial foi preenchida com as informações metodológicas relevantes para este estudo (acima citadas).

A análise estatística dos dados foi composta pelo teste do sinal para comparar proporções, bem como, em decorrência do baixo número de casos observados (estudos encontrados [n=6]), foi empregado o teste exato do Fisher para analisar possíveis associações. Todas as análises foram efetuadas em software estatístico, bem como, o nível de significância foi pré-fixado em $\mathrm{p}<5 \%$.

\section{RESULTADOS}

Dentre todos sete trabalhos detectados em nosso levantamento, um foi excluído por ser um estudo de revisão. Todos os seis trabalhos que cumpriam os critérios de inclusão foram publicados em revistas relacionadas à pediatria $(n=1 ; 16,6 \%), \quad$ medicina/saúde coletiva $(n=2 ;$ $33,2 \%)$, medicina do esporte $(n=2 ; 33,2 \%)$ e fisioterapia ( $n=1 ; 16,6 \%)$. A Tabela 1 apresenta os trabalhos selecionados, bem como, os seus temas.

Tabela 1. Estudos envolvidos na revisão de literatura.

\begin{tabular}{|c|c|c|c|}
\hline Autor & Revista & Ano & Tema \\
\hline Sguizzatto et al. & $\begin{array}{l}\text { São Paulo Medical } \\
\text { Journal }\end{array}$ & 2006 & QV em atletas idosas \\
\hline Silva et al. & $\begin{array}{l}\text { Ciência \& Saúde } \\
\text { Coletiva }\end{array}$ & 2010 & $\begin{array}{l}\text { Analise da QV em diferentes indivíduos: } \\
\text { Professores, funcionários e estudantes }\end{array}$ \\
\hline Alencar et al. & $\begin{array}{l}\text { Fisioterapia } \\
\text { movimento }\end{array}$ & 2010 & QV em idosas ativas e sedentárias \\
\hline Toscano e Egypto & $\begin{array}{l}\text { Revista Brasileira de } \\
\text { Medicina do Esporte }\end{array}$ & 2009 & $\begin{array}{l}\text { Nível de AF e QV relacionada com a saúde de } \\
\text { idosas }\end{array}$ \\
\hline Menêses et al. & $\begin{array}{l}\text { Revista Brasileira de } \\
\text { Medicina do Esporte }\end{array}$ & 2011 & QV de indivíduos com Claudicação Intermitente \\
\hline Gordia et al. & $\begin{array}{l}\text { Revista Paulista de } \\
\text { Pediatria }\end{array}$ & 2010 & $\begin{array}{l}\text { Domínio psicológico da qualidade de vida de } \\
\text { adolescentes }\end{array}$ \\
\hline
\end{tabular}

QV - qualidade de vida

A maioria dos estudos encontrados foi publicada nos dois últimos anos ( $n=4 ; 65 \%)$. Da mesma forma, embora não de maneira significativa estatisticamente $(p=0,404)$, houve maior ocorrência de estudos envolvendo idosos (Figura 1). Apenas 33\% dos estudos nacionais encontrados analisaram populações adultas ou pediátricas.

Os resultados encontrados demonstraram grande similaridade no que se refere ao instrumento de medida da qualidade de vida utilizado (Figura 2). Apenas dois instrumentos foram utilizados para quantificar a qualidade de vida, o questionário SF-36 e o WHOQOL.

Metade dos estudos encontrados envolveram homens em suas amostras $(n=3)$ e, nenhum estudo, analisou homens somente. Por outro lado, em todos os estudos encontrados, houve mulheres nas amostras.

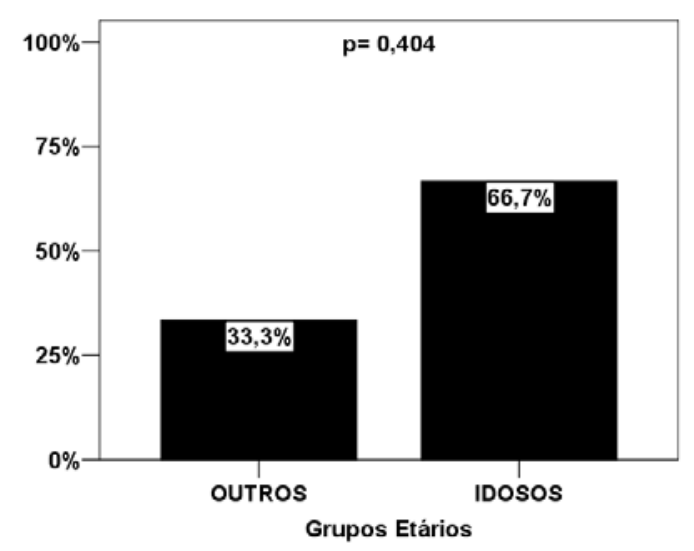

Figura 1. Artigos publicados sobre qualidade de vida segundo grupos etários estudos. 


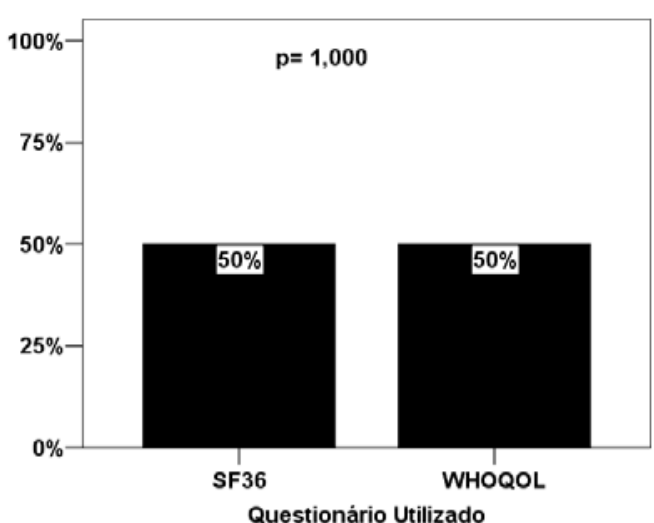

Figura 2. Instrumentos utilizados para avaliar qualidade de vida.

O tamanho das amostras envolvidas nos estudos variou de 30 a 863 sujeitos. Em todos os estudos analisados ( $n=6 ; 100 \%)$, houve interação entre maior prática de atividades físicas e melhores escores nos domínios da qualidade de vida.

\section{DISCUSSÃO}

Estudo de revisão conduzido com estudos nacionais presentes na base SCIELO o qual identificou seis estudos originais envolvendo o relacionamento entre qualidade de vida e prática de atividades físicas. Houve predomínio de dois tipos de questionários utilizados, o SF-36 e o WHOQOL. A análise dos anos de publicação dos artigos selecionados indica que, nos últimos anos, houve um importante aumento no interesse pelo assunto. Por outro lado, embora com esse aumento expressivo, algumas limitações precisam ser apontadas.

A grande maioria dos estudos foi conduzida com idosos. Analisando atletas, o estudo de Sguizzatto et al. (2006) comparou a qualidade de vida e a presença de sintomas depressivos entre mulheres de 60 anos ou mais, 16 atletas e 15 sedentárias saudáveis (sem queixas ou sintomas de dores). Os resultados obtidos identificaram diferença significante nas capacidades funcionais, dor, estado de saúde mental e nos escores de depressão (melhor para as ativas). Similarmente, Alencar et al. (2010) compararam idosas (não-atletas) ativas e sedentárias e identificaram resultados similares. Tais achados indicam que a atividade física regular pode proporcionar menos sintomas depressivos entre idosas.

Tal interesse excessivo por populações idosas cria uma distorção ao não considerar a possível interação entre estas variáveis em outros grupos etários. Nesse sentido, Gordia et al. (2010) analisaram 608 adolescentes entre 14 e 20 anos. A qualidade de vida foi avaliada pelo WHOQOL e para avaliar a atividade física foi usado o questionário internacional de atividade física (IPAQ). Os autores concluíram que as intervenções voltadas para a atividade física podem contribuir para a melhoria do domínio psicológico da qualidade de vida de adolescentes.

A principal limitação do estudo reside no fato de apenas o SCIELO ter sido consultada. Sabe-se que outros estudos sobre o tema, inclusive nacionais, podem ter sido publicados em revista internacionais não indexadas no SCIELO. Assim, futuros estudos deveriam envolver outras bases de dados.

Em resumo, os achados da presente revisão de literatura indicam que a qualidade de vida parece estar associada positivamente com a maior prática de atividades físicas, independente da idade. Porém, ressalta-se que mais estudos devem ser conduzidos com outros grupos etários, que não idosos.

\section{CONFLITO DE INTERESSE}

Os autores declaram não haver qualquer potencial conflito de interesse que possa interferir na imparcialidade deste trabalho científico.

\section{REFERÊNCIAS}

Alencar NA, Souza Junior JV, Aragão JCB, Ferreira MA, Dantas E. Nível de atividade física, autonomia funcional e qualidade e vida em idosas 
ativas e sedentárias. Rev Fisioterapia Mov. 2010; 23(3):473-81.

Gordia AP, Silva RCR, Quadros TMB, Campos W. Variáveis comportamentais e sociodemográficas estão associadas ao domínio psicológico da qualidade de vida de adolescentes. Rev Paulista Pediat. 2010; 28(1):29-35. http://dx.doi.org/10.1590/S0103$\underline{05822010000100006}$

Meneses $A L$ et al. Relação entre aptidão física e os indicadores de qualidade de vida de indivíduos com Claudicação intermitente. Rev Bras Med Esporte. 2011;17(3):175-8.

Sguizzatto GT, Garcez-Leme LE, Casimiro L. Evaluation of the quality of life among elderly female athletes. São Paulo Med J. 2006;124(5):304-5.

http://dx.doi.org/10.1590/S1516-

$\underline{31802006000500014}$

Silva RS, Silva I, Silva RA, Souza L, Tomasi E. Atividade física e qualidade de vida. Ciênc \& $\begin{array}{lll}\text { Saúde Col. 2007;15(1):115-20. } & \text {. }\end{array}$ http://dx.doi.org/10.1590/S1413-

$\underline{81232010000100017}$

Toscano JJO, Egypto EP. Qualidade de Vida em Idosos com Distintos Níveis de Atividade Física. Rev Bras Med Esporte. 2009; 15(3):169-73. 